\title{
CONFLICTIVA SECULARIZACIÓN: SOBRE SOCIOLOGÍA, RELIGIÓN E HISTORIA ${ }^{1}$
}

\section{CONFLICTIVE SECULARIZATION: ON SOCIOLOGY, RELIGION AND HISTORY}

\author{
Julio de la Cueva Merino \\ Universidad de Castilla-La Mancha
}

Entregado el 9-1-2015 y aceptado el 4-6-2015.

\begin{abstract}
Resumen: En los últimos treinta años, el paradigma de la secularización como modelo explicativo del cambio religioso en la modernidad ha estado sometido a intensa contestación y debate. Mientras que un número no desdeñable de sociólogos sigue manteniendo su validez, una cantidad cada vez mayor de estudiosos critican su inadecuación y proponen modelos alternativos que ayuden a explicar la sostenida presencia de la religión en las sociedades modernas. Este artículo pretende revisar el estado de este debate, que se desarrolla principalmente en el ámbito de la sociología, y contribuir a un mejor conocimiento del mismo entre los historiadores. Asimismo, se propone poner de relieve las posibilidades de reflexión para la historia religiosa y la historia en general que ofrecen las controversias actuales sobre la secularización. Por último, reivindica la utilidad del concepto secularización en el contexto español, siempre que esta se entienda como una «secularización conflictiva», una
\end{abstract}

1 Este trabajo ha sido elaborado en el marco de los proyectos de investigación HAR2011-29383-C02-01 y PPII-2014-020-P, financiados, respectivamente, por el Ministerio de Economía y Competitividad y por la Junta de Comunidades de Castilla-La Mancha. Deseo expresar mi agradecimiento a Joseba Louzao, por haberme animado a actualizar mis conocimientos sobre sociología de la secularización, y a Francisco José Carmona, por su generosidad en leer atentamente mi manuscrito y hacerle cuidadosas y acertadas observaciones. 
secularización problematizada en su conceptualización y en su desarrollo historiográfico.

Palabras clave: secularización, laicización, modernidad, historia religiosa, secularización conflictiva.

\begin{abstract}
For the last thirty years the suitability of the secularization paradigm as an explanatory model of religious change in modernity has been the object of intense debate. Whereas many sociologists still defend its validity, many more criticize its adequacy and propose alternative models in order to account for the sustained presence of religion in modern societies. This paper aims at revising the current state of this debate, which takes place mostly in the field of sociology, and at contributing to its knowledge among historians. Furthermore, it proposes to highlight that some of these controversies have an interest in order to expand our instruments for interpretation of religious history and general history. Last, the article advocates the usefulness of the concept «secularization» within the Spanish context, secularization understood as «conflictive secularization», secularization problematized in its definition an its historiographical developments.
\end{abstract}

Key words: secularization, laicization, modernity, religious history, conflictive secularization. 
Desde la Ilustración, una de las autorrepresentaciones más exitosas de la modernidad ha sido la de considerarse a sí misma incompatible con cualquier forma de religión que no fuera tendencialmente residual. Las razones argüidas para diagnosticar la progresiva pérdida de pertinencia social e individual de la religión han sido diversas y, en cierto modo concomitantes: el avance de la racionalidad científica y del progreso material, la prospectiva liberación del hombre de las cadenas de frustración y opresión que lo esclavizan y alienan, los procesos de urbanización, industrialización y societalización que inducen la ruptura de los vínculos con las creencias y lealtades propias de las sociedades tradicionales.

Hasta un pasado reciente, esta narrativa secular parecía hallar constatación universal en los hechos observables. La religión, en efecto, perdía su tradicional presencia en la esfera pública. Además, su retirada de los ámbitos anteriormente ocupados por las ideologías, instituciones y personas que la representaban se veía acompañada, en no pocos países y ocasiones, de un largo conflicto de la Iglesia o las Iglesias con los Estados nacidos de las revoluciones liberales de los siglos XVIII y XIX y con los regímenes alumbrados por las revoluciones socialistas del siglo XX. A veces incluso, la salida de la religión se acompañaba del surgimiento coetáneo de nuevas religiones «seculares» o de no menos nuevas religiones «políticas» ${ }^{2}$. En cualquier caso, la definitiva confirmación de la bondad interpretativa y predictiva de lo que daría en llamarse «teoría» o «tesis» o «paradigma» de la secularización provino de las espectaculares cifras de abandono de la práctica religiosa en las décadas posteriores a la segunda guerra mundial y, muy particularmente, a partir de los años sesenta. De manera nada sorprendente por tanto, fue precisamente durante lo años sesenta y setenta del siglo pasado cuando este paradigma alcanzó su máximo desarrollo académico.

El éxito de la tesis de la «secularización» ha sido tal que se ha convertido en el prisma a través del cual observa el presente y el futuro de la religión no sólo una minoría de académicos, intelectuales o militantes laicistas, sino también el conjunto de la opinión pública occidental. Más aún, desde la categoría de «secularización», han diseñado sus políticas religiosas - o la ausencia de las mismas - buena parte de los gobiernos occidentales y sus estrategias pastorales, asimismo, buena parte de las con-

${ }^{2}$ Un magnífico ejemplo de historia religiosa de Europa que toma como hilo argumental la secularización, la de René Rémond, Religion et société en Europe: Essai sur la sécularisation des sociétés européennes aux XIXe et XXe siècles (1789-1998), Seuil, París, 1998. 
fesiones religiosas - al menos, las denominaciones judeocristianas - . Sin embargo, ciertas percepciones están cambiando desde los años ochenta. Las religiones no acaban de desaparecer; antes bien, en vastas extensiones del planeta parecen más presentes que nunca. Y algunos de quienes antes extendían su certificado de defunción, entonan ahora la palinodia y denuncian la vieja tesis secularizadora como un mito. Recordemos los casos del célebre sociólogo austro-norteamericano, padre de la moderna teoría de la secularización, Peter Berger, o del sociólogo y teólogo estadounidense, próximo a la llamada «teología de la muerte de Dios», Harvey $\mathrm{Cox}^{3}$.

El propósito de estas páginas es dar cuenta de las recientes transformaciones en el paradigma de estudio del cambio religioso contemporáneo en el terreno de las ciencias sociales - fundamentalmente, en el ámbito de la sociología ${ }^{4}-$ y apuntar una meditación sobre cómo estas pueden afectar a la historia como disciplina. No pretendo ser enteramente original ni, mucho menos, ocultar mi deuda con otros historiadores que me han precedido en la reflexión: Joseba Louzao, más recientemente, en un trabajo de singular y enorme valor; Manuel Álvarez Tardío y Gregorio Alonso, hace más tiempo, en trabajos verdaderamente pioneros ${ }^{5}$. Mi ensayo trata, como los suyos, de dar a conocer un debate todavía, en buena medida, desconocido para la historiografía no especializada y de llegar al final, si es posible, a alguna conclusión de mediana plausibilidad. He señalado que la secularización ha proporcionado el instrumento a través del cual muchos han contemplado y siguen contemplando el presente y el futuro de la religión. Acabo de adelantar también que este enfoque está dejando de ser he-

3 Peter Berger, «The Desecularization of the World. A Global Overview», en Peter Berger (ed.), The Desecularization of the World. Resurgent Religion and World Politics, Ethics and Public Policy Center, Washington, D.C., 1999, pp. 1-18; Harvey Cox, «The Myth of the Twentieth Century. The Rise and Fall of Secularization», en Gregory Baum (ed.), The Twentieth Century. A Theological Overview, Geoffrey Chapman, Londres, 1999, pp. 135-143.

${ }^{4}$ Y más específicamente en el ámbito de la sociología francesa y anglosajona, aunque realizaré algunas referencias a la sociología de la religión en lengua castellana y algún apunte sobre la contribución habermasiana al debate.

5 Joseba Louzao Villar, «La recomposición religiosa en la modernidad: un marco conceptual para comprender el enfrentamiento entre laicidad y confesionalidad en la España contemporánea», Hispania Sacra, 60, 2008, pp. 331-354; Manuel Álvarez Tardío, «Política y secularización en la Europa contemporánea», Studia Historica. Historia Contemporánea, 16, 1998, pp. 143-166; Gregorio Alonso, «La secularización de las sociedades europeas», Historia Social, 46, 2003, pp. 137-157. 
gemónico en el campo de la sociología, al compás de recientes cambios en las realidades y percepciones de la presencia pública y privada de las religiones. A la luz de estos acontecimientos actuales, a nosotros, historiadores, se nos plantea cómo podemos y debemos contemplar el pasado religioso. Si las preocupaciones del presente, de cada presente, nunca han sido ajenas a la tarea del investigador, sin lugar a dudas el presente cambiante de la religión en la sociedad y en la sociología interpela las interpretaciones que realizamos sobre el lugar de la religión en la historia.

\section{La revancha de Dios: el retorno contemporáneo de lo religioso}

Casi desde el mismo momento de la reformulación moderna de la tesis de la secularización en los años sesenta y setenta del pasado siglo se alzaron voces críticas con la pertinencia de la misma - entre ellas, algunas luego tan conocidas como las del teórico de la secularización David Martin- ${ }^{6}$. En aquellas circunstancias de modernidad rampante y contrastado declive religioso, tales voces pasaron en buena medida desapercibidas.

Por esos años, el desafío más evidente a la tesis de que el mundo se secularizaba como consecuencia del imparable avance del proceso de modernización procedía del caso norteamericano. De hecho, en la medida en que es difícil negar la modernidad de los Estados Unidos de América, el apego de los norteamericanos a sus creencias y sus prácticas religiosas constituía, y para muchos sigue constituyendo, el principal reto a la tesis de la secularización ${ }^{7}$. Más aún, el panorama religioso estadounidense ma-

6 David Martin, «Towards eliminating the concept of secularization», en Julius Gould (ed.), Penguin Survey of the Social Sciences, Penguin, Baltimore, 1965, pp. 169-182; Larry Shiner, «The Concept of Secularization in Empirical Research», Journal for the Scientific Study of Religion, 6, 1967, pp. 207-220; Andrew Greeley, El hombre no secular. Persistencia de la religión, Cristiandad, Madrid, 1974 (1972); David Bell, «The return of the sacred?», British Journal of Sociology, 28, 1977, pp. 419-490. Los clásicos de David Martin sobre la secularización: David Martin, A General Theory of Secularization, Oxford, Basil Blackwell, 1978, y On Secularization: Towards a Revised General Theory, Ashgate, Aldershot, 2005.

7 Roger Finke, «An Unsecular America», en Steve Bruce (ed.), Religion and Modernization. Sociologists and Historians Debate the Secularization Thesis, Clarendon Press, Oxford, 1992, pp. 143-169; Peter Berger, Grace Davie y Effie Fokas, Religious America, Secular Europe? A Theme and Variations, Ashgate, Aldershot, 2008. Sin embargo, ciertas voces niegan la excepcionalidad norteamericana: Steve Bruce, Secularization: in defence of an unfashionable theory, Oxford University Press, Oxford, 2011, pp. 157-176. 
nifestaba no sólo la persistencia de las iglesias preexistentes, sino la vitalidad aportada por fórmulas como la llamada religión contracultural, los Nuevos Movimientos Religiosos y las religiones de importación.

En cualquier caso, la vitalidad religiosa de la sociedad estadounidense en los años sesenta y setenta podía pasar medianamente desapercibida para el mundo académico - así como para el gran público occidental atento a los medios de comunicación - hasta que otros acontecimientos pusieron de manifiesto la sostenida o recuperada importancia, ya no meramente privada sino pública, de lo religioso. En 1977, se producía la primera gran victoria de los partidos religiosos en Israel, que acababa con casi cuarenta años de gobiernos laicos de izquierda en el Estado judío. En 1978, Juan Pablo II era elegido Papa y ponía en marcha su programa de segunda evangelización. En 1979, el triunfo de la revolución islámica en Irán revelaba al mundo una nueva presencia militante del Islam, que no ha cejado hasta ahora. Estos son los hechos que ponía de relieve Gilles Kepel en su famosa obra La revancha de Dios. Judios, cristianos y musulmanes a la reconquista del mundo, publicada en 19918; pero igualmente podría haber señalado otros: el impulso dado por la Teología de la Liberación a la triunfante revolución nicaragüense y a otros movimientos revolucionarios o reivindicativos en América Latina; la identidad firmemente católica del sindicato polaco Solidaridad, que constituyó el más potente y a la postre exitoso movimiento popular de oposición al socialismo real; la influencia creciente del protestantismo conservador en el diseño de la política norteamericana. La lista sería demasiado extensa y debería incluir, asimismo, referencias a los espacios donde las religiones, transmutadas en identidades, han provocado $-\mathrm{y}$, en algunos casos, continúan provocando- enfrentamientos armados a distintas escalas: Irlanda del Norte, Yugoslavia, el Próximo y Medio Oriente, el subcontinente indio, diversas zonas de África.

En fin, los sangrientos atentados del 11 de septiembre de 2001 en Nueva York y Washington, el resto de ataques islamistas anteriores y posteriores - incluidos los de Madrid - , las guerras de Afganistán, Irak y Siria y la emergencia del Estado Islámico, han contribuido definitivamente a que la ya existente conciencia de la importancia del factor religioso en la política y geopolítica mundial se universalice y profundice. Sin salir del

${ }^{8}$ Gilles Kepel, La revancha de Dios. Cristianos, judios y musulmanes a la reconquista del mundo, Anaya-Mario Muchnik, Madrid, 1991 (1991). 
ámbito académico, ha destacado, por su fuerza mediática, el debate sobre el enfrentamiento entre civilización cristiano-occidental y civilización islámica promovido por el polémico libro de Samuel Huntington, El choque de civilizaciones - publicado por primera vez en inglés en 1996- que algunos han entendido que bien pudiera haberse titulado «el choque de religiones» ${ }^{9}$. Como quiera que sea y de una u otra forma, nunca antes en los últimos cien años había preocupado tanto la cuestión religiosa a profesionales de la comunicación, políticos, politólogos y sociólogos en los planos nacional e internacional.

Y es que, como señala José Casanova en otro de los libros fundamentales de los últimos años, Religiones públicas en el mundo moderno, desde finales de los años setenta se asiste a un proceso de «desprivatización» de la religión. Es decir, que «las tradiciones religiosas de todo el mundo se niegan a aceptar el papel marginal y privatizado que les han reservado las teorías de la modernidad y las teorías de la secularización» ${ }^{10}$. En realidad, la mayoría de las tradiciones religiosas nunca habrían aceptado, o sólo a regañadientes, su relegación a la esfera privada; pero su negativa a aceptar esta consecuencia del proceso de secularización - cuando no el proceso en sí-, se habría hecho mundialmente visible sobre todo en la década de los ochenta. Como asimismo indica Rafael Díaz-Salazar, más allá de las creencias de cada uno, es inútil empeñarse en proclamar que la religión es un asunto privado: ésta siempre ha sido, y continúa siendo, una «cuestión pública» ${ }^{11}$. Es, en buena medida, este marco de una «sociedad postsecular» el que impulsó al filósofo alemán Jürgen Habermas a irrumpir en el debate político-sociológico sobre la religión y proponer que es en interés del propio Estado liberal que «se permita el libre acceso de las voces religiosas tanto en la esfera público-política como en la participación política de las organizaciones religiosas» ${ }^{12}$.

9 Samuel P. Huntington, El choque de civilizaciones y la configuración del orden mundial, Paidós, Barcelona, 2005 (1996); Georges Corm, La cuestión religiosa en el siglo XXI. Geopolítica y crisis de la modernidad, Taurus, Madrid, 2007 (2006).

10 José Casanova, Religiones públicas en el mundo moderno, PPC, Madrid, 2000 (1994), p. 18.

11 Rafael Díaz-Salazar, Democracia laica y religión pública, Taurus, Madrid, 2007.

12 Jürgen Habermas, Entre naturalismo y religión, Paidós Ibérica, Barcelona, 2006 (2005), p. 117. Sobre el término «sociedad postsecular», popularizado por Habermas, él mismo señala que lo toma de su compatriota el sociólogo Klaus Eder. Una discusión de la reflexión habermasiana sobre la religión en Craig Calhoun, Eduardo Mendieta y Jonathan Van Antwerpen, Habermas and Religion, Polity Press, Londres, 2013. 
Como quiera que sea, parecen ser bastantes los indicadores de que, señala Peter Berger, «el mundo es hoy tan furiosamente religioso como siempre y en algunos lugares, más que nunca ${ }^{13}$. En ese sentido, el sociólogo norteamericano, dando por periclitada la vigencia de la teoría que él mismo contribuyó a enunciar, proponía una nueva fórmula interpretativa del proceso de cambio religioso vigente en la actualidad: la «desecularización» del mundo. Aun así, el propio Berger admitiría dos espacios de excepción a tal «desecularización». El primero sería un espacio deslocalizado, habitado por intelectuales y profesionales con educación occidental, los cuales constituirían una elite internacional secular, pequeña pero muy influyente. Un segundo espacio coincidiría con el continente europeo, único territorio donde la tesis de la secularización ha podido probar empíricamente su consistencia. Es por ello que se podría afirmar que la secularización europea no constituye ya la regla, sino una auténtica excepción: un resultado más $-\mathrm{y}$ bastante peculiar-, entre otros muchos posibles, de la modernidad en materia religiosa.

Incluso así, algunos sociólogos se plantean que quizá también Europa esté dejando de ser excepcional. Los viejos dioses resisten, y nuevos dioses vienen a instalarse en nuestro territorio de la mano de la globalización y - como parte de este fenómeno - de la mano de los inmigrantes asentados en nuestro suelo y procedentes del Este del continente, de África, de Asia y de América Latina.

Por un lado, pues, los viejos dioses permanecen en el corazón de muchos europeos, quienes en su mayoría mantienen creencias religiosas, aunque ya no crean lo que creían ni practiquen lo que practicaban sus mayores, ni se sometan a las autoridades religiosas a las que estos se sometían ${ }^{14}$. Se trata del fenómeno que Grace Davie ha denominado believing without belonging («creer sin pertenecer») ${ }^{15}$. Más aún, pese a la indivi-

${ }^{13}$ P. Berger, «The Desecularization of the World», op. cit. Véase también para lo que sigue, Peter Berger, «Secularization and de-secularization», en Linda Woodhead (ed.), Religions in the Modern World, Routledge, Londres, 2002, pp. 291-298 y Grace Davie, Europe: The Exceptional Case. Parameters of Faith in the Modern World, Darton, Longman and Todd, Londres, 2002.

${ }^{14}$ Rafael Díaz-Salazar, «La religión vacía. Un análisis de la transición religiosa en Occidente», en Rafael Díaz-Salazar, Salvador Giner y Fernando Velasco (eds.), Formas modernas de religión, Alianza, Madrid, 1994, pp. 71-114.

15 Grace Davie, Religion in Britain since 1945. Believing without Belonging, Blackwell, Oxford, 1994. La tesis ha sido cuestionada por David Voas y Alasdair Crockett, «Religion in Britain: Neither Believing nor Belonging», Sociology, 39, 2005, pp. 11-28. 
dualización innegable de la religiosidad europea, las instituciones religiosas continúan aguantando el temporal secularizador, como la propia Davie ha argumentado: ya sea integrándose en el sector voluntario de la sociedad - en paralelo a otras asociaciones y ONGs - , ya sea sirviendo de religión vicaria - es decir, procurando un «hilo de memoria» con el pasado y ofreciendo servicios religiosos a la carta, fundamentalmente para sancionar los ritos de paso-; pero también haciendo oír sus voces, cada vez con menos reservas, en la arena pública ${ }^{16}$. En fin, la religión - fundamentalmente, todavía, la cristiana - continúa informando en buena medida la cultura, el pensamiento y las mentalidades de los europeos ${ }^{17}$.

Por otro lado, también, nuevos dioses están llegando a Europa con la globalización. Una parte de ellos recaban la adhesión de los europeos a través de las denominadas «sectas», dando lugar, junto a las viejas religiones renovadas, a un «panorama de creencias no menos rico y activo que aquel de las sociedades de las que se considera - con o sin razónque hunden todavía sus raíces en el universo de la tradición» ${ }^{18}$. Nuevas deidades viajan, asimismo, en el equipaje de los inmigrantes que, por millones, se han establecido en el Viejo Continente durante los últimos cincuenta años: todo el panteón hindú, diferentes versiones del Dios cristiano y, sobre todo, Alá, bajo cuya sombra protectora el Islam se está convirtiendo en la segunda religión practicada en Europa. De esta manera y por todo ello, la propia consideración de Europa como un territorio secularizado, o al menos irreversiblemente secularizado, parece cada vez más dudosa.

El ejemplo de Francia ilustra magníficamente las dificultades actuales por las que atraviesa la secularidad europea. Es Francia, probablemente, el país occidental donde la doble lógica de la secularización sociológica y la laicización política ha avanzado más e, históricamente, antes ${ }^{19}$. La laicización política francesa ha dado lugar a un modelo de

${ }^{16}$ Grace Davie, Religion in Modern Europe. A Memory Mutates, Oxford University Press, Oxford, 2000, y «The persistence of institutional religion in modern Europe», en Linda Woodhead, Paul Heelas y David Martin (eds.), Peter Berger and the Study of Religion, Routledge, Londres, 2001, pp. 101-111. Lo del «hilo de memoria» en Danièle Hervieu-Léger, La religión, hilo de memoria, Herder, Barcelona, 2005 (1993).

17 Danièle Hervieu-Léger, La religion en miettes ou la question des sectes, CalmannLévy, París, 2001, p. 10.

18 Ibidem, p. 9.

19 Jean-Louis Ormières, L'Europe désenchantée. La fin de l'Europe chrétienne? France, Belgique, Espagne, Italie, Portugal, Fayard, París, 2005. 
regulación de las relaciones entre las Iglesias y el Estado conocido con el nombre de «laicidad». La laicidad republicana actual proviene de la implícitamente desarrollada en la Ley de Separación de las Iglesias y el Estado de 1905 y de la explícitamente consagrada en las Constituciones francesas de 1946 y 1958. Esta laicidad francesa, un día considerada por propios y extraños como corolario político perfecto y necesario del proceso de secularización y ejemplo a imitar por el resto del mundo, es hoy en día, sin embargo, por usar las palabras de Danièle Hervieu-Léger, una laïcité en panne, una «laicidad averiada» ${ }^{20}$, una laicidad que muestra sus deficiencias ante las nuevas realidades de finales del siglo XX y principios del XXI. ¿Cuáles son esas realidades? La profundización ultramoderna del individualismo cívico y la desvalorización postmoderna del discurso racionalista, las cuales llevan a una devaluación del propio ideario y ética laica republicana; la transformación de los estados contemporáneos en estados neocorporativos, en cuyo seno los grupos de interés son habitualmente requeridos por las autoridades para que participen en la toma de decisiones; la multiculturalización de la sociedad francesa y la mutación de las religiones en identidades que exigen un reconocimiento, en particular por parte del Islam ${ }^{21}$. De esta manera, se ha abierto un debate en Francia - sobre todo en torno al centenario de la Ley de Separación - y han sido muchas las voces, también desde el campo de las ciencias sociales, que han propuesto un nuevo pacto laico, una laicización de la propia laicidad; es decir, una revisión del régimen de laicidad y su evolución hacia una laicidad «mediadora», «de reconocimiento», «plural» ${ }^{22}$.

20 Danièle Hervieu-Léger, La religion en mouvement. Le pèlerin et le converti, Flammarion, París, 1999, p.241.

21 Jean Baudoin y Philippe Portier «La laïcité française. Approche d'une métamorphose», en Jean Baudoin y Philippe Portier (dirs.), La laïcité, un valeur d'aujord'hui?, Presses Universitaires de Rennes, Rennes, 2001, pp. 15-34; Marcel Gauchet, La religión en la democracia. Los caminos del laicismo, El Cobre-Editorial Complutense, Barcelona-Madrid, 2003 (1998); Philippe Portier, «De la séparation à la reconnaisance. L'évolution du régime français de laïcité», en Jean-Robert Armogathe y Jean-Paul Willaime (dirs.), Les mutations contemporaines du religieux, Brepols, Turnhout, 2003, pp. 1-24.

${ }^{22}$ D. Hervieu-Léger, La religion en mouvement, op. cit.; Jean-Paul Willaime, Le retour du religieux dans la sphère publique: Vers une laïcité de reconnaissance et de dialogue, Olivétan, París, 2008; Jean Baubérot y Micheline Milot, Laïcité sans frontières, Seuil, París, 2011. 


\section{Dios ha muerto: el paradigma de la secularización}

«Secularización: RIP». De manera tan provocativa participaba en el año 2000 el paso a mejor vida de la vieja tesis -y de la realidad que pretendía explicar - uno de sus principales adversarios, el sociólogo norteamericano Rodney Stark ${ }^{23}$. Dos años más tarde, Steve Bruce, uno de los más tenaces defensores del viejo modelo anunciaba de manera no menos provocadora, aunque poco original, la vitalidad del proceso secularizador, y titulaba un libro suyo Dios ha muerto ${ }^{24}$. El debate entre partidarios y detractores del modelo explicativo de la secularización está muy vivo ${ }^{25}$. Y aunque en la actualidad la moda sociológica ${ }^{26}$ parezca decantarse más del lado de los segundos, se hace necesario caracterizar, lo que, hasta hace bien poco, ha constituido conventional sociological wisdom ${ }^{27}$.

Este lugar común se resiste, sin embargo, a las definiciones comúnmente aceptadas. Un punto de coincidencia entre los diversos téoricos del llamado modelo «heredado» $\mathrm{u}$ «ortodoxo» ${ }^{28}$ tal vez sea la consideración de que «la modernidad conlleva un declive de la religión» ${ }^{29}$. Harvey Cox simplificaría este vínculo asemejándolo a una especie de «ecuación de suma cero»: «cuanta más modernización, menos religión» (y viceversa) ${ }^{30}$.

${ }^{23}$ Rodney Stark, «Secularization: R.I.P.», en William H. Swatos Jr. y Daniel W. A. Olson (eds.), The Secularization Debate, Rowman \& Littlefield, Lanham (Maryland), 2000, pp. 42-66.

${ }^{24}$ Steve Bruce, God is Dead. Secularization in the West, Blackwell, Oxford, 2002.

25 Dos buenos ejemplos de volúmenes que recogen posiciones contrastadas: W. H. Swatos Jr. y D. V. A. Olson (eds.), The Secularization Debate, op. cit., y «After Secularization», número doble especial de The Hedgehog Review, 8 (1-2), 2006 (http://www.iasc-culture.org/THR/hedgehog_review_2006-Spring-Summer.php).

26 Steve Bruce reconocía, irónicamente, en el título de su último libro cuán demodées podían sonar sus tesis en defensa del paradigma de la secularización: Secularization, op. cit.

27 Frank J. Lechner, «The Case Against Secularization: A Rebuttal», Social Forces, 69, 1991, pp. 1103-1119.

28 «Heredado», en Bryan Wilson, «Secularization: The Inherited Model», en Phillip E. Hammond (ed.), The Sacred in a Secular Age. Toward Revision in the Scientific Study of Religion, University of California Press, Berkeley-Los Ángeles, 1985, pp. 9-20; «ortodoxo», en Roy Wallis y Steve Bruce, «Secularization: The Orthodox Model», en Steve Bruce (ed.): Religion and Modernization. Sociologists and Historians Debate the Secularization Thesis, Clarendon Press, Oxford, 1992, pp. 8-30.

${ }_{29}$ Peter L. Berger, The Many Altars of Modernity. Toward a Paradigm for Religion in a Pluralist Age, Boston-Berlín, Walter de Gruyter, 2014, p. IX.

30 Cox, «The Myth», op. cit. 
La veracidad de esta ecuación ha sido sostenida, de una u otra forma y de manera más o menos convincente, por multitud de pensadores, académicos e intelectuales desde el siglo XVIII ${ }^{31}$.

Aunque el modelo sociológico de la secularización conoció su edad dorada en las décadas posteriores a la Segunda Guerra Mundial, sus fundamentos ideológicos y teóricos habían sido establecidos con indudable anterioridad. Se haría interminable - además de imposible - mencionar todos los estudiosos que, desde el siglo XVIII hasta mediados del XX, han relacionado modernidad y secularización como variables interdependientes. Podrían, no obstante, subrayarse algunos nombres entre quienes constituyeron el canon «prebélico» de la tesis de la secularización. Obligado sería comenzar por la figura señera de Auguste Comte, representativa del sentir de un buen número de pensadores ilustrados y decimonónicos para los cuales el progreso de la razón, la técnica y el espíritu científico inevitablemente socavarían los fundamentos de la creencia religiosa, la cual en la sociedad futura no tendría sentido o sería sustituida por algún tipo de religión positivista o secular. Tampoco se podría dejar de señalar la aportación de Karl Marx, quien, haciéndose eco de otras voces - notablemente la de Ludwig Feuerbach-, consideraba la religión como un síntoma de la alienación humana, como un expediente de enmascaramiento de la explotación económica y como una parte de esa superestructura ideológica de la sociedad destinada irremisiblemente a desaparecer cuando el desarrollo de las fuerzas productivas entrase en conflicto con las relaciones de producción y provocase el cambio revolucionario del modo de producción.

La nómina de teóricos prebélicos no estaría completa sin la figura capital de Max Weber. Con Weber, la teoría de la secularización abandonaba la «prehistoria»y entraba en su propia «historia» 32 . Fue, de hecho, el académico alemán el primero en emplear el término secularización en su sentido sociológico. Lo hizo en 1904-1905 en su Ética protestante y el

31 Véanse los trabajos de Olivier Tschannen, Les théories de la sécularisation, Droz, Ginebra, 1992; William H. Swatos Jr. y Kevin J. Christiano, «Secularization Theory: The Course of a Concept», en Swatos Jr. y Olson (eds.), The Secularization Debate, op. cit., pp. 1-20; Gregory Baum, Religion and Alienation. A Theological Reading of Society, Novalis-Orbis Books, Ottawa-Maryknoll (Nueva York), 2006; Jean-Claude Monod, Sécularisation et laïcité, PUF, París, 2008; Rob Warner, Secularization and Its Discontents, Continuum, Londres-Nueva York, 2010; Francisco J. Carmona Fernández: «Cristianismo, laicismo y laicidad», en Francisco J. Carmona Fernández (coord.), Historia del cristianismo, IV. El mundo contemporáneo, Trotta, Madrid, 2010, pp. 670-750.

32 Tschannen, Les théories, op. cit. 
espíritu del capitalismo, aunque sin mucho énfasis, pues Weber prefería y popularizó otra expresión: «el desencantamiento del mundo». Curiosamente Weber no despreciaba el papel de la religión en la conformación de la modernidad. Antes bien, como es sabido, valoraba mucho la importancia del cristianismo protestante en el surgimiento del capitalismo. La valoración del factor religioso en la génesis de la modernización no le impedía, sin embargo, vislumbrar - y no sin sentimiento - que el moderno proceso de racionalización conducía a un declive de la presencia social de la religión. Últimamente, las teorías de Weber han sido reformuladas en Francia por el filósofo Marcel Gauchet, en particular en su exitoso libro El desencantamiento del mundo. Una historia política de la religión ${ }^{33}$. Empleando una terminología que ha obtenido gran aceptación, Gauchet piensa la historia religiosa como la historia del tránsito de una religión estructurante de todo lo humano a una religión residual, sin trascendencia social. A este proceso lo llama «salida de la religión» y al cristianismo, la «religión de la salida de la religión»:

No es Gauchet el único deudor de Weber. Lo son todos los integrantes del paradigma de la secularización, un paradigma que se convirtió en tal al alcanzar el consenso de la comunidad sociológica entre finales de los años cincuenta y principios de los setenta. Olivier Tschannen, cuya reconstrucción de los avatares del paradigma nos es esencial aquí, identifica ocho autores a quienes se les puede considerar «portadores» del mismo: Peter Berger, Thomas Luckmann, Bryan Wilson, David Martin, Richard Fenn, Karel Dobbelaere, Talcott Parsons y Robert Bellah. De estos ocho «clásicos», el propio Tschannen destaca el magisterio de Berger y Wilson, autores de los dos manifiestos fundacionales del paradigma, dos libros publicados ambos en los años sesenta y cuyos títulos respectivos fueron vertidos al castellano como El dosel sagrado, en el caso de la obra de Berger, y La religión en la sociedad (en inglés, Religion in Secular Society), en el caso de la de Wilson. Asimismo, subraya Tschannen la importancia del trabajo de Karel Dobbelaere, cuyo amplio estado de la cuestión de 1981 resultó fundamental para la consolidación del paradigma ${ }^{34}$.

${ }_{33}$ Marcel Gauchet, El desencantamiento del mundo. Una historia política de la religión, Trotta-Universidad de Granada, Madrid-Granada, 2005 (1985).

${ }^{34}$ Peter Berger, El dosel sagrado. Elementos para una sociología de la religión, Amorrortu, Buenos Aires, 1971 (1967); Bryan Wilson, La religión en la sociedad, Labor, Barcelona, 1969 (1966); Karel Dobbelaere, «Secularization: A Multidimensional Concept», Current Sociology, 31 (2), 1981, pp. 3-217. 
Quizá no sorprenda, a estas alturas, la precisión de que ambos padres fundadores mencionados, Peter Berger y Bryan Wilson, hayan matizado posteriormente sus posturas respecto de la secularización. En el caso de Berger, ya se ha señalado que, más que matizar sus hallazgos anteriores, el sociólogo estadounidense de origen austriaco ha imprimido un cambio diametral a su reflexión. En sus estudios iniciales, la paulatina racionalización del mundo y, sobre todo, el pluralismo religioso moderno conducían necesariamente a la secularización, mientras que en trabajos más recientes insiste en la desecularización de la sociedad global ${ }^{35}$. En el caso de Wilson, su obra posterior ha insistido en uno de los aspectos ciertamente ya apuntados en sus primeros trabajos: que por secularización entiende la pérdida de significación social de la religión, es decir, que la religión deja de ser significativa para el funcionamiento del sistema social, aunque los individuos puedan - y ahí es donde ahora introduce su reserva - continuar siendo religiosos ${ }^{36}$. Ha dejado, pues, Wilson para alguno de sus discípulos más enragés la defensa del modelo secularizador en su totalidad, incluido «el declive de la extensión de la práctica religiosa por parte de los individuos, de las creencias religiosas de cualquier tipo y de las conductas acordes con tales creencias religiosas» ${ }^{37}$.

De esta manera, se entra en una cuestión clave, sin la cual no se concibe el meollo del paradigma de la secularización, ni tampoco se comprenden las controversias suscitadas en torno a la adecuación de sus propuestas interpretativas a la realidad social: tal cuestión consiste en que por secularización no se entiende una, sino varias cosas. Esto es lo que Dobbelaere caracterizó, en su influyente y ya citado trend report de 1981, como «multidimensionalidad» del concepto de secularización. En 2002, reeditó su estado de la cuestión con algunas modificaciones terminológicas. Así, Dobbelaere distingue, básicamente, tres dimensiones o niveles del concepto de secularización. El primer nivel sería el macronivel societal e implicaría tanto la pérdida por parte de la religión de su papel totalizador y la asunción por parte de otras instituciones sociales de funciones antes desempeñadas por la religión, como la desacralización o desencan-

35 Berger, «The desecularization», op. cit., y «Secularization», op. cit.

36 Bryan Wilson, Religion in sociological perspective, Oxford University Press, Oxford, 1982; «Secularization», op. cit., y «Reflections on a Many Sided Controversy», en Steve Bruce (ed.), Religion and Modernization. Sociologist and Historians Debate the Secularization Thesis, Clarendon Press, Oxford, 1992, pp. 195-210.

37 Bruce, God is Dead, op. cit., p. 9. 
tamiento del mundo. El segundo se identificaría con el mesonivel organizacional y haría referencia a los cambios y adaptaciones a la modernidad que se producen en el interior de las propias confesiones religiosas. El tercero, por último, correspondería a un micronivel individual e incluiría el declive del compromiso personal con la religión ${ }^{38}$.

\section{El peregrino y el converso: la recomposición religiosa en la modernidad}

La multiplicidad de sentidos del término «secularización» nos pone sobre la pista de alguna de sus debilidades. Cuando una palabra quiere decir tantas cosas, es difícil que alguien no busque otro término, concepto, modelo, que resulte más concreto y significativo. Algo de eso hay en las dos principales alternativas que hoy en día se proponen para rellenar el vacío que la insatisfacción con la tesis de la secularización produce: la teoría de la elección o decisión racional aplicada a la religión y la propuesta interpretativa de la recomposición religiosa en la modernidad. Ambos casos no constituyen, en opinión de quien suscribe estas líneas, «enmiendas a la totalidad» del paradigma de la secularización, sino intentos de adecuación del mismo a las realidades concretas del mundo occidental actual.

De ambas alternativas hablaré a continuación. Sin embargo, previamente será necesario llamar la atención sobre el hecho de que el principal desafío lanzado en la actualidad sobre el paradigma de la secularización no proviene seguramente de estas nuevas (re-)construcciones teóricas. Procede, más bien, del cuestionamiento del segundo término del binomio al que la secularización, como he señalado, habitualmente va unida: la modernidad. Excedería el propósito de estas páginas, dar cuenta de un debate paralelo al de la secularización e indudablemente de singular significado para éste: el debate sobre la modernización. En cualquier caso, resulta insoslayable recordar aquí algo que ya he apuntado: que desde la cada vez más extendida perspectiva intercultural y postcolonial en las ciencias humanas y sociales resulta difícil seguir defendiendo la existencia de «modelos»-modelos prescritos por Occidente, siempre se entiende - que serían de obligado tránsito en todo el mundo. En ese sentido,

38 Dobbelaere, «Secularization», op. cit., y Secularization: An Analysis at Three Levels, PIE-Peter Lang, Bruselas, 2002. 
la «modernidad», en la medida en que sea un fenómeno global, asume diversas formas y ritmos en diferentes sociedades en todo el planeta e incluso, a veces, en el interior de una misma sociedad. Esta es la tesis de las «múltiples modernidades», formulada por Shmuel Eisenstadt en su famoso artículo seminal publicado en $2000^{39}$.

Ciñéndonos, de nuevo, al más familiar terreno de la secularización, la afirmación de la existencia de una pluralidad de modernidades conllevaría, asimismo, la afirmación de la existencia de una pluralidad de respuestas religiosas al reto de la modernización ${ }^{40}$. La secularización sería una de las respuestas, la propia de Occidente y - más característicamente- de Europa. Más aún, incluso asumiendo que modernización y secularización sean de alguna forma dos caras de la misma moneda, se debería conceder que, al igual que se dan «múltiples modernidades», existirían igualmente «múltiples secularidades», «múltiples secularizaciones», múltiples recorridos y resultados del proceso de secularización ${ }^{41}$.

Pero antes de llegar al continente europeo, seguramente convenga dar un rodeo por Norteamérica, exponiendo la propuesta alternativa a la secularización que ha conocido un mayor éxito entre las elaboradas en los Estados Unidos: la aplicación de la teoría de la elección racional (Rational Choice Theory) a la religión. Esta teoría parte de una serie de principios que se pueden enunciar de la siguiente manera: los individuos actúan racionalmente - es decir, maximizando beneficios y minimizando costes - , las necesidades de los individuos no varían demasiado de persona a persona y en el seno de las sociedades se alcanza un equilibrio como resultado de la agregación e interacción de las necesidades individuales. El modelo que se aplica, pues, es el del mercado donde interactúan productores y consumidores y que tiende siempre a un equilibrio ${ }^{42}$. A partir de estos supuestos, un grupo de sociólogos norteamericanos, liderado

39 S. N. Eisenstadt, «Multiple modernities», Daedalus, 129, 2000, pp. 1-29.

40 Yves Lambert, «Religion in Modernity as a New Axial Age: secularization or New Religious Forms», en Swatos Jr. y Olson (eds.), The Secularization Debate, op . cit., pp. 95-125; Linda Woodhead, «Studying religion and modernity», en Linda Woodhead (ed.), Religion in the modern world, Routledge, Londres, 2002, pp. 1-12.

${ }^{41}$ Monika Wohlrab-Sahr y Marian Burchardt, «Multiple Secularities: Toward a Cultural Sociology of Secular Modernities», Comparative Sociology, 11, 2012, pp. 875-909: Martin, On Secularization, op. cit.

${ }^{42}$ Laurence R. Iannaconne: «Rational Choice. Framework for the Scientific Study of Religion», en Lawrence A. Young (ed.), Rational Choice Theory and Religion. Summary and Assessment, Routledge, Nueva York, 1997, pp. 25-45. 
por Rodney Clark, ha elaborado una teoría de la religión que pretende dar razón científica de la vitalidad religiosa estadounidense y posicionarse abierta y militantemente en contra de la tesis de la secularización ${ }^{43}$.

Stark y sus asociados parten del supuesto de que los seres humanos siempre buscan recompensas y evitan costes. Hay determinadas recompensas que nunca han dejado de anhelar los humanos y que no pueden obtenerse de manera inmediata: la inmortalidad, un sentido para la vida. La única fuente plausible para este tipo de recompensas - a veces también llamadas compensaciones - sólo podría situarse en el ámbito de lo sobrenatural. En ese sentido, las religiones serían organizaciones dedicadas, principalmente, a proporcionar compensaciones basadas sobre presupuestos sobrenaturales. Como los seres humanos insisten en sus deseos de compensación sobrenatural, la cantidad de religión en una sociedad permanece relativamente constante. Por tanto, la secularización o declive religioso es sólo una de las tendencias existentes en todas las economías de la religión, siempre contrarrestada en los mercados religiosos por los fenómenos paralelos de la revitalización o revival religioso - nuevos grupos y formas de religiosidad en el seno de las iglesias existentes - y de la innovación religiosa - nuevas religiones-. Dentro de esta lógica de mercado, para estos sociólogos norteamericanos, el excepcional progreso de la secularización en Europa se explicaría no por el lado de la demanda - ausencia de demanda religiosa por parte de los individuos - , sino de la oferta - organizaciones religiosas ineficientes dentro de economías religiosas altamente reguladas - . La ausencia de auténtico pluralismo religioso en Europa sería la causa de su aparente secularización: los individuos no encontrarían en el mercado religioso productos especializados que se ajusten a sus necesidades ${ }^{44}$.

${ }^{43}$ Rodney Stark y William Sims Bainbridge, The Future of Religion. Secularization, Revival and Cult Formation, University of California Press, Berkeley-Los Ángeles, 1985, y A Theory of Religion, Lang, Nueva York, 1987; Young (ed.), Rational Choice, op. cit.; Rodney Stark y Roger Finke, ): Acts of Faith. Explaining the Human Side of Religion, University of California Press, Berkeley-Los Ángeles, 2000. El primer reconocimiento de la emergencia de este nuevo paradigma en R. Stephen Warner. «Work in Progress Toward a New Paradigm for the Sociological Study of Religion in the United States», American Journal of Sociology, 98, 1993, pp. 1044-1093. La crítica del paradigma en Steve Bruce, Choice and Religion. A Critique of Rational Choice Theory, Oxford University Press, Oxford, 1999.

${ }^{44}$ Rodney Stark y Laurence Iannaconne, «A Supply-Side Reinterpretation of the "Secularization" of Europe», Journal for the Scientific Study of Religion, 33, 1994, pp. 230252 . 
Llegamos así, pues, finalmente a Europa, territorio de la excepcionalidad, una excepcionalidad que, como he adelantado, está siendo también objeto de matización por parte de bastantes académicos del Viejo Continente. De esa manera, se encontraría en construcción una tentativa de interpretación que, para autores como Frédéric Lennoir, se ha constituido ya en auténtico paradigma alternativo al de la secularización - o a su reverso, la desecularización - ${ }^{45}$. Este nuevo paradigma sería el de la «recomposición de lo religioso en la modernidad» ${ }^{46}$. También es común referirse a este fenómeno como «individualización de la religión» e incluso hallar en el clásico de Thomas Luckmann de 1967, La religión invisible, un primer exponente de esta manera de comprender el cambio religioso en la modernidad, al entender el sociólogo que la «secularización» consiste fundamentalmente en una «privatización» de la religión (aunque esta adscripción haya sido criticada, por ejemplo, por Ulrich Beck, que entienden que «individualización» no es lo mismo que «privatización», pues la primera sería compatible con un reforzamiento de la presencia pública de las religiones) ${ }^{47}$.

De cualquier forma, no sería hasta finales de los años ochenta y principios de los noventa cuando la propuesta interpretativa de la «recomposición religiosa» comenzase a asentarse como paradigma interpretativo alternativo. Aunque algunos de sus promotores se ubiquen en las Islas Británicas, como es el caso de Grace Davie, sus principales exponentes desarrollan su actividad académica en Francia. Lennoir sería uno de ellos y él mismo ofrece el nombre de otros tres sociólogos con el fin de completar el cuadro de los padres del nuevo paradigma: Yves Lambert, Danièle Hervieu-Léger y Jean-Paul Willaime.

En principio, ni Lambert ni Hervieu-Léger ni Willaime niegan la realidad del proceso de secularización o de «salida de la religión» en Europa. Sin embargo, aceptan que éste se debe entender, en esencia, como un proceso de pérdida - o descomposición-, pero también de recomposición de lo religioso. Para ello se inspiran en una fórmula de François-André Isambert, quien preconizaba la comprensión de la secularización como

45 Frédéric Lenoir: Las metamorfosis de Dios. La nueva espiritualidad occidental, Alianza, Madrid, 2003.

${ }^{46}$ Louzao Villar, «La recomposición religiosa», op. cit.

47 Ulrich Beck, El Dios personal: la individualización de la religión y el «espíritu» del cosmopolitismo, Paidós, Barcelona, 2009 (2008). Thomas Luckmann, La religión invisible: El problema de la religión en la sociedad moderna, Sígueme, Salamanca, 1973 (1967). 
«mutación religiosa de la sociedad $»^{48}$. El primer estudio empírico que evidenció la veracidad de la intuición de Isambert fue probablemente una investigación de Yves Lambert sobre la religiosidad bretona, la cual concluía que lo religioso, más que desaparecer en Bretaña $-\mathrm{y}$ por extensión en Francia - a lo largo del siglo xx, había simplemente cambiado en sus maneras de sentirse y manifestarse ${ }^{49}$.

Sin embargo, tal vez hayan sido Danièle Hervieu-Léger y Jean-Paul Willaime quienes hayan profundizado de manera más original en las relaciones entre la modernidad tardía y la pervivencia transformada de lo religioso en ella. La consagración del individualismo por parte de la modernidad es seguramente el punto de entronque de las visiones de ambos sociólogos y, sobre todo en el caso de Hervieu-Léger, el elemento nodal de su comprensión de la recomposición de la creencia en la Europa actual. En sus primeros ensayos, esta autora contemplaba ya una modernidad racionalista y humanista, que alimentaba en el individuo la utopía de la autorrealización ilimitada y que, de esta manera, lo empujaba paradójicamente a situarse en un plano eminentemente religioso. En obras posteriores ha insistido en el hecho de que, también en la sociedad moderna, el individuo necesita dotar de sentido y continuidad a su experiencia vital y que las creencias religiosas siguen demostrando su competencia para llevar a cabo esa tarea ${ }^{50}$. Lo característico de la modernidad, sin embargo, sería el protagonismo del individuo $-\mathrm{y}$ no de la institución religiosa - a la hora de elegir y dar contenido a las creencias y prácticas que le convienen y satisfacen. De esta forma, la religión en la modernidad tardía adoptaría la forma de una religión en movimiento, cuyas figuras características serían el peregrino y el converso. El primero escogería creencias ajustadas a su propia experiencia a través de operaciones de bricolaje religioso, dando lugar a una religiosidad a la carta, muy fluida e incierta en sus pertenencias comunitarias. El converso, por su parte, se adheriría a una comunidad religiosa, sí, pero como elección individual y autónoma, pues en la modernidad una identidad religiosa auténtica sólo puede ser una iden-

48 Danièle Hervieu-Léger, «Sécularisation et modernité religieuse», Esprit, octubre 1985, pp. 50-62.

49 Yves Lambert, Dieu change en Bretagne. La religion de 1900 à nos jours, Cerf, París, 1985.

${ }^{50}$ Hervieu-Léger, «Sécularisation», op. cit.; Danièle Hervieu-Léger y Françoise Champion, Vers un nouveau christianisme? Introduction à la sociologie du christianisme occidental, Cerf, París, 1986; Hervieu-Léger, La religion en miettes..., op. cit. 
tidad elegida $-\mathrm{y}$ no impuesta o transmitida $-{ }^{51}$. Algunas de estas identidades serían verdaderas etnorreligiones, a las cuales se puede - dando la vuelta al hallazgo semántico de Grace Davie - incluso «pertenecer sin creer» ${ }^{52}$.

Para Jean-Paul Willaime, por su parte, no cabe duda sobre la realidad del proceso secularizador - principalmente, sobre la pérdida de autoridad de las instituciones religiosas sobre los individuos y la sociedad-; es decir, no cabe duda sobre los efectos disolventes de la modernidad sobre la religión. Pero tampoco vacila el sociólogo sobre la existencia de fenómenos de recomposición de lo religioso en la ultramodernidad ${ }^{53}$. Porque Willaime prefiere emplear el término «ultramodernidad» - en vez de postmodernidad, por ejemplo - , para designar la fase de radicalización y universalización de las consecuencias de la modernidad. Esta radicalización se manifestaría en la extensión de los efectos sociales de dos de los principios constitutivos de la modernidad: la reflexividad y la individualización. Por mor del primero, la modernidad se vuelve autorreflexiva y aprende a criticar sus propios mitos y utopías, incluida los generados por la ciencia y la política. Como consecuencia del segundo, el individuo termina por rechazar cualquier magisterio, del tipo que sea. La ultramodernidad sería, pues, una modernidad desencantada, problematizada, autorrelativizada. Pues bien, en el seno de esta modernidad autosecularizada, y que pierde por tanto sus caracteres de «religión secular», lo religioso se abre su propio camino. No es un retorno de la religión antigua, una especie de revancha de ésta contra la modernidad, sino - insiste - se trata de una recomposición de lo religioso en el seno de una sociedad secularizada. La modernidad no sería, pues, el fin de lo religioso, sino lo religioso «de otra manera» ${ }^{54}$.

En fin, este apartado no quedaría completo sin una referencia a la ultimísima de las grandes tesis alternativas a la secularización, y esta ha sido muy recientemente formulada por Peter Berger. Esta nueva propuesta bebe, además, a mi entender, en buena medida, de las dos anteriormente

${ }^{51}$ Hervieu-Léger, La religion en mouvement, op. cit.

${ }^{52}$ Hervieu-Léger, La religión, op. cit., p. 266. Olivier Roy ha dado una vuelta más a estos argumentos en La santa ignorancia: el tiempo de la religión sin cultura, Península, Barcelona, 2010.

53 Jean-Paul Willaime, Sociologie des religions, PUF, París, 1995.

54 François Boespflug, Françoise Dunand y Jean-Paul Willaime, Pour une mémoire des religions, La Découverte, París, 1996; Jean-Paul Willaime, Europe et religions. Les enjeux du XxIe siècle, Fayard, París, 2004. 
expuestas, así como de las propias reflexiones de Berger sobre el pluralismo religioso, presentes en su obra desde el inicio. Ya se ha señalado cómo Berger, al renegar ruidosamente -él mismo reconoce el ruidoen 1999 del paradigma del que fuera valedor, propuso que se comprendiese la conspicua presencia contemporánea de la religión en términos de «desecularización». Ciertamente, esta era una descripción del estado de la religión en el mundo actual, pero apenas podía equivaler a un nuevo paradigma que sustituyese al que pretendía desafiar. La propuesta paradigmática de Berger ha llegado quince años después en su libro The Many Altars of Modernity. Como ya apunta el título, y más claramente aún el subtítulo - Toward a Paradigm for Religion in a Pluralist Age-, para Berger la gran novedad de la modernidad en términos de religión no es la «secularización», sino el «pluralismo», que no se debe confundir con aquella. Tal pluralismo - que no simplemente pluralidad - implicaría no sólo la coexistencia de diferentes religiones, sino la coexistencia de lo religioso y lo secular en el seno de las sociedades y en el interior de los propios individuos ${ }^{55}$. Volveré a ello más adelante.

\section{La secularización conflictiva: historizando el debate}

Con el fin de avanzar en el debate en torno a la secularización, José Casanova ha puesto de manifiesto la necesidad de «historizar» las categorías, teorías y narrativas de las que se sirven los estudiosos para interpretar los procesos de cambio religioso. Para el sociólogo, «historizar» sería «globalizar», es decir, pensar los fenómenos en una dimensión mundial que impida la distorsión siempre latente a la generalización a partir de un caso particular - por importante que éste parezca $-{ }^{56}$. Interpretando a Casanova, bien se podría entender, pues, que su «historizar-como-globa-

55 Berger, The Many Altars, op. cit.

56 José Casanova, «Beyond European and American Exceptionalisms: towards a Global Perspective», en Grace Davie, Pauk Heelas y Linda Woodhead (eds.), Predicting Religion. Christian, Secular and Alternative Futures, Ashgate, Aldershot, 2003, pp. 1729. Esta exigencia de «historización» ya había sido formulada antes por Philip S. Gorski, «Historicizing the Secularization Debate: Church, State, and Society in Late Medieval and Early Modern Europe, ca. 1300 to 1700», American Sociological Review, 65, 2000, pp. 138-167, y «Historicizing the Secularization Debate», en Michele Dillon, Handbook of the Sociology of Religion, Cambridge University Press, Cambidge, 2003, pp. 110-122, http://dx.doi.org/10.1017/CBO9780511807961.009. 
lizar» significa en buena medida «historizar-como-singularizar»; es decir, considerar la singularidad de los casos, algo que, por otro lado, siempre hemos hecho los historiadores. Desde una perspectiva histórica -insiste Casanova-, la serie de cambios que conocemos con el nombre de secularización evidenciarían una dinámica singular a una clase muy particular de régimen religioso: la Cristiandad occidental en sus formulaciones católicas y protestantes. En este sentido, coincide con el filósofo Charles Taylor, quien, en su monumental y compleja A Secular Age propone un estudio genealógico de la secularidad ceñido a Occidente, entendido este como «Cristiandad latina», un estudio donde la secularización no se concibe si no es en términos históricos ${ }^{57}$. La secularización occidental $-\mathrm{y}$ particularmente la europea - , pues, constituiría un proceso particular, singular y probablemente irrepetible, y no, por tanto, un modelo teleológico que revele al resto del mundo un futuro universalmente necesario. Otras realidades habrán de estudiarse según sus propios parámetros, aunque la secularización continúe proporcionando un marco analítico imprescindible para la investigación comparada.

La secularización sería, pues, un fenómeno predominantemente occidental y, sin embargo, tampoco Occidente procura un modelo ni cerrado ni único. Sobre el cierre e irreversibilidad del modelo de la secularización, en las páginas precedentes se han aducido razonamientos sociológicos por los cuales se puede considerar que el último capítulo del proceso de cambio religioso occidental no se ha escrito todavía. En cuanto a la unicidad o diversidad de modelos de secularización, ya se ha aludido a la perenne contraposición de la excepcionalidad europea versus la excepcionalidad americana en la literatura sociológica. No obstante, se debe añadir que, asimismo, ha sido habitual en sociología la identificación de diferentes modelos y ritmos de secularización en el interior de la propia Europa. Estas distinciones han venido no sólo de parte de sociólogos con una aproximación matizada a la tesis de la secularización como David Martin, sino incluso del lado de fervientes defensores de la «ortodoxia» teórica como Steve Bruce ${ }^{58}$. De tal manera, se han intentado diversas tipologías que se han resuelto en distinciones entre sociedades de predominio católico, sociedades de predominio protestante, sociedades protestantes con plu-

57 Charles Taylor, A Secular Age, Harvard University Press, Cambridge (Massachusetts), 2007.

58 Martin, A General Theory, op. cit., y On Secularization, op. cit.; Wallis y Bruce, «Secularization», op. cit. 
ralismo religioso, sociedades duales..., cada uno de los tipos correspondiéndose con diferentes desarrollos del proceso secularizador y diferentes resultados del mismo, al menos en lo relativo a la presencia pública y estatuto jurídico de las religiones.

Hablar de distintas vías hacia un régimen religioso de secularidad en Occidente implica también hablar de diferentes cadencias temporales. La secularización, en la medida que demos por buena su realidad, no puede ser un proceso lineal, progresivo y unívoco sino un proceso histórico inscrito en circunstancias históricas, particulares y cambiantes, sujeto a altibajos, sobresaltos, avances y retrocesos, sometido a períodos de estancamiento y etapas de aceleración, marcado también por las respuestas y reacciones de las confesiones y los creyentes ${ }^{59}$. Un proceso cuyo devenir, ritmo y resultado final - si es que se puede decir tal cosa en historia- no se encuentra desde luego escrito. Y si no se halla escrito es porque la secularización no puede entenderse como consecuencia natural y directa de la modernización, un desarrollo que se genera automáticamente a partir de determinadas condiciones socioeconómicas y se desenvuelve de manera igualmente mecánica.

Si dejamos de considerar la secularización solo como un proceso dependiente de meras variables socioeconómicas y ajeno, por tanto, a la agencia humana, entonces su estudio puede comenzar a implicarnos más como historiadores. A este efecto resultará útil traer a colación la diferencia establecida por Jean Baubérot y Françoise Champion entre «secularización» y «laicización». En palabras del historiador y sociólogo francés, la «secularización» constituiría «un proceso de pérdida progresiva y relativa de pertinencia social de lo religioso, que tiene lugar a nivel de tendencias profundas, principalmente por el juego de la dinámica social, sin mayor confrontación entre las esferas de lo político y lo religioso». En contraste, la «laicización» tendría más que ver con «las tensiones explícitas entre diferentes fuerzas sociales (religiosas, culturales, políticas, incluso militares) que pueden tomar la forma de un conflicto abierto». Según Baubérot, en los procesos de laicización, «lo que está en juego es, por tanto, el control del aparato del Estado (o, al menos, una fuerte influencia sobre el mismo) para que dé (incluso imponga) soluciones relativas a la

59 Véanse, por ejemplo, los trabajos de Hugh McLeod «Secular Cities? Berlin, London and New York in the Later Nineteenth an the Earlier Twentieth Centuries», en Bruce (ed.), Religion and Modernization, op. cit, pp. 59-89, y Secularisation in Western Europe 18481914, Macmillan, Londres, 2000. 
religión como institución social» ${ }^{60}$. Sería posible, incluso — añado yo-, no limitar la consideración del conflicto a una lucha por la dominación de los aparatos de control estatal, pues la disputa abarcaría y se resolvería en otros muchos terrenos. En cualquier caso, lo que se produce es una disputa política, por la distribución del poder. Particularmente interesante para nosotros como historiadores habrá de ser examinar en qué medida y de qué manera la actividad de los agentes que se posicionan a favor o en contra de la laicización contribuyó de manera efectiva al progreso o contención de la pérdida de pertinencia social de lo religioso.

Nos encontramos, en cualquier caso, ante un rasgo fundamental de la secularización: su naturaleza esencialmente conflictiva. Un rasgo, de nuevo, particularmente interesante para la ciencia histórica. Esta conflictividad afecta sin duda a los países donde los procesos de «laicización» han marcado la pauta, como es el caso de los países católicos de la Europa latina y la América latina. Un caso que ha llevado a Jean-Pierre Bastian a definir una especie de «latinidad» religiosa, conformada por sociedades que se caracterizarían, de un lado, por la existencia de un catolicismo tradicionalmente hegemónico y, de otro, por la aparición más reciente de una modernidad de ruptura con el mismo. La oposición entre tradición católica y modernidad secular se habría traducido en un agudo conflicto entre Iglesia y Estado ${ }^{61}$. La conflictividad, empero, también se manifiesta en los espacios donde la «secularización» tranquila parece haber predominado. Así lo ha mostrado, por ejemplo, Christian Smith en su obra The Secular Revolution, sobre la secularización de la vida pública norteamericana, en la que afirma que ésta «no fue el producto natural, inevitable y abstracto de la modernización, sino el resultado de una lucha entre grupos enfrentados con intereses encontrados en busca del control sobre el conocimiento

60 Prácticamente con las mismas palabras tanto en Jean Baubérot, «Laïcité, laïcisation, sécularisation», en Alain Dierkens (ed.), Pluralisme religieux et laïcités dans l'Union européennne, Université de Bruxelles, Bruselas, 1994, pp. 9-17, como en «Sécularisation et laïcisation», en Benoît Pellistrandi (ed.), L'histoire religieuse en France et en Espagne, Madrid: Casa de Velázquez, pp. 17-38. También maneja esta distinción Françoise Champion, «Les rapports Église-État dans les pays européens de tradition protestante et de tradition catholique: essai d'analyse», Social Compass, 40, 1993, pp. 589-609.

61 Jean-Pierre Bastian, «Introducción. Aprender a descentrar la mirada sobre la modernidad religiosa», en Jean-Pierre Bastian (coord.), La modernidad religiosa. Europa latina y América latina en perspectiva comparada, Fondo de Cultura Económica, México, 2004. 
y las instituciones.» ${ }^{62}$ Se vea como quiera verse, la secularización ni pudo ser ni es un proceso sociológico abstracto, sino un campo de disputa política y cultural, donde individuos, grupos y movimientos laicizadores y contrasecularizadoras se confrontan, estimulándose mutuamente en sus avances y retrocesos.

Acabo de afirmar la secularización como «campo de disputa política y cultural» y me detendré unas líneas sobre lo segundo. No será necesario insistir sobre la importancia de lo cultural en la historiografía desde hace cuarenta años. No sorprenderá, por tanto, que uno de los ámbitos que se revela más fructífero para los estudios históricos sobre los procesos de cambio religioso sea éste, el de la cultura, el lenguaje y el discurso. Y, de nuevo, en su versión conflictiva, pues no suele constituir la cultura un espacio de encuentro sino un campo de batalla. En este sentido, resulta muy apropiada la ya clásica propuesta del sociólogo norteamericano James D. Hunter de denominar «guerra de culturas» o «guerra cultural» (culture war) al tipo de enfrentamiento que se alimenta, fundamentalmente, de la sustentación de cosmovisiones ideológicas, políticas, sociales y morales diferentes y que se dirime, también básicamente, en el amplio terreno de la cultura. Precisamente Culture Wars se titulaba una obra colectiva editada hace unos años y que adoptaba y adaptaba tal concepto al análisis de la confrontación entre secularismo y catolicismo, anticlericalismo y clericalismo, en la Europa del siglo XIX ${ }^{63}$. Además, desde la historia cultural se ha demostrado posible la realización de aportaciones significativas por parte de historiadores al debate sociológico sobre la religión. Tal es el notable caso, por ejemplo, del libro de Callum Brown The Death of Christian Britain, en el que se plantea una revisión completa del paradigma de la secularización a partir de la historia cultural y de género. La secularización de Gran Bretaña - y la de otros países europeos, Australia y Nueva Zelanda - se habría producido abruptamente a partir de la década de 1960 y habría tomado la forma de una auténtica revolución cultural relacionada

${ }^{62}$ Christian Smith, «Introduction: Rethinking Secularization of American Public Life», en Christian Smith (ed.), The Secular Revolution. Power, Interests and Conflicts in the Secularization of American Public Life, University of California Press, Berkeley-Los Ángeles, 2003, pp. 1-96.

63 James Davison Hunter, Culture War. The Struggle to Define America, Basic Books, Nueva York, 1991; Christopher Clark y Wolfram Kaiser (eds.), Culture Wars. Secular-Catholic Conflict in Nineteenth-Century Europe, Cambridge University Press, Cambridge, 2003. Una y otra obra han pasado bastante desapercibidas en nuestro país, con excepciones como las de Louzao, «La recomposición religiosa», op. cit., que hace suya la propuesta interpretativa. 
con la incapacidad de las Iglesias cristianas de seguir generando discursos propios capaces de competir exitosamente con los poderosos discursos seculares de los swinging sixties. En el avance de la secularización habría sido de particular importancia la impotencia eclesiástica para mantener una formulación de la identidad femenina en términos de piedad y moralidad cristiana frente a las pujantes propuestas alternativas de liberación secular de la mujer ${ }^{64}$.

Los tres primeros apartados del presente artículo se han dedicado a dar cuenta de las crecientes dificultades del paradigma de la secularización, puesto en entredicho en el reciente debate sociológico. Y, sin embargo, estas modestas propuestas para uso de historiadores responden a la necesidad de ofrecer interpretaciones históricas de los procesos contemporáneos de cambio religioso mediante el manejo crítico de una versión matizada de dicho paradigma. ¿Qué ocurre, entonces, con todo lo prolijamente expuesto en torno al contemporáneo retorno de lo sagrado, la desecularización del mundo, la pluralidad de modernidades o la recomposición de lo religioso en la modernidad? ¿Podemos considerarlo pertinente? $\mathrm{Y}$, en ese caso, ¿cómo afecta al quehacer historiográfico?

Ya se ha visto que la pertinencia de cuestionar la inexorabilidad universal de la secularización no parece, en absoluto, fuera de lugar. Al menos, no lo consideran así reputados sociólogos, algunos de ellos padres del propio paradigma de la secularización. Disponemos, además, de la evidencia desplegada ante nuestros ojos por parte de politólogos, demógrafos, estadísticos y periodistas. No insistiré más en ello, pues ya se ha discutido con amplitud en las páginas precedentes. En cualquier caso y a mi parecer, todo ello nos incumbe desde luego como historiadores y nos implica de diversas maneras. Me centraré en dos que me parecen fundamentales.

En primer lugar, la actualidad e intensidad del debate sociopolítico y sociológico sobre el retorno o la permanencia de la religión debería ayudarnos a despejar cualquier duda sobre la importancia de la atención historiográfica que demanda esta materia, ya sea como un campo de estudio propio - llámese «historia religiosa» o de cualquier otro modo-, ya sea como un objeto ordinario de la historia política, social y cultural. La consecuencia necesaria habría de ser la definitiva normalización historiográfica del estudio de la religión, algo particularmente urgente en la historiografía

${ }^{64}$ Callum G. Brown, The Death of Christian Britain. Understanding Secularisation, 1800-2000, Routledge, Londres, 2009. 
española, tan tradicionalmente desdeñosa de este aspecto. Siempre ha existido una razón obvia para que los historiadores nos ocupemos de la religión: que ésta constituye un factor sin el cual no puede explicarse ni comprenderse el pasado. En la coyuntura actual, es posible añadir un motivo suplementario: la religión como fenómeno individual y colectivo no da signos de estar abocada a la extinción en un futuro próximo, sino que parece más bien llamada a continuar desempeñando un papel de protagonismo social en absoluto menor. Nos dejemos llevar por los desafíos del presente o nos movamos por las inquietudes del pasado, la religión y cuanto la rodea -incluidos los conflictos que genera o que se generan a su alrededor - no pueden sino constituir un terreno privilegiado de interés historiográfico.

En segundo lugar, los historiadores podríamos sentirnos interpelados por los debates sociológicos sobre la religión de un modo más sutil. Si nos dejamos cuestionar por éstos, será difícilmente sostenible que en nuestros análisis continuemos dando automáticamente por descontado que la secularización es consecuencia universal y directa de la modernidad. Es decir, tal vez deberíamos replantearnos el presupuesto de que sólo hay una forma de estar en la «modernidad» y que esa forma ha sido siempre, en todo lugar y en todo momento, la estricta «secularidad». Sin abandonar el ámbito de Occidente - sin salir incluso de las fronteras de Europa-, si algo pone de manifiesto la investigación histórica es la importancia continuada de las iglesias y de las creencias religiosas hasta, al menos, los años sesenta del siglo XX. También entre la población urbana. También entre buena parte de las clases trabajadoras. También, desde luego, entre las mujeres y no como mera muestra fehaciente del retraso de su emancipación respecto de la de los varones ${ }^{65}$. En ese sentido, la historiografía no puede ignorar ni minusvalorar los fenómenos de permanencia de las confesiones o, incluso, de revitalización religiosa que caracterizan la modernidad europea y que han llevado a algunos historiadores a conceptuar - a partir de una expresión acuñada por Olaf Blaschke ${ }^{66}$ - la etapa compren-

65 Véase Inmaculada Blasco Herranz, «Género y religión: de la feminización de la religión a la movilización católica femenina. Un revisión crítica», Historia Social, 53, 2005 , pp. 119-136.

${ }^{66}$ La producción historiográfica de Olaf Blaschke se encuentra mayoritariamente en alemán. En inglés se puede acceder a una síntesis de sus planteamientos sobre la «segunda edad confesional» en el primer capítulo de Offenders or Victims? German Jews and the Causes of Modern Catholic Antisemitism, The Hebrew University of Jerusalem-University of Nebraska Press, Jerusalén-Lincoln (Nebraska), 2009. 
dida entre 1830 y 1960 como una «segunda edad confesional» frente a la tradicional asunción de que toda la contemporaneidad habría meramente de tratarse como una «era de secularización».

En relación a esta revitalización de lo religioso, cabría preguntarse también si ciertos elementos de los mencionados procesos de recomposición religiosa resultan exclusivos de la modernidad tardía o si algunas de sus características se encuentran ya con anterioridad. Así, por ejemplo, la fe reencontrada del «converso» y su fuerte sentido comunitario de pertenencia se reconocerá en el creciente militantismo confesional frente a otras denominaciones o, más frecuentemente en el mundo católico latino, frente al avance de la secularidad. Pero, por otro lado, aspectos de la religión individualizada del «peregrino» podrían identificarse en la efervescencia de manifestaciones de la religiosidad popular ${ }^{67}$, los movimientos piadosos que eclosionan en los siglos XIX y XX, la fe en nuevas creencias como el espiritismo y la teosofía, o el nacimiento de otras disidencias religiosas, entre las que podría contarse en el caso español el particular caso de la espiritualidad krauso-institucionista ${ }^{68}$. Además, y en el mismo sentido, el historiador habrá de tener en cuenta que la religiosidad individual, incluida la de los católicos, siempre - y con más razón en las sociedades modernas - es resultado, en mayor o menor medida, de un procesos de «bricolaje» personal o «personalización» de la fe, la cual no se conforma necesariamente en todos los puntos a la estricta ortodoxia o a la común ortopraxis de la iglesia a la que se pertenece ${ }^{69}$. Más aún, la «fluida construcción» de fe y secularidad en el interior de los individuos, la operatividad del discurso secular también en la conciencia de los creyentes, el relativismo introducido por el pluralismo en todos los

67 Hace más de treinta años Thomas Kselman (Miracles and Prophecies in Nineteenth Century France, Rutgers University Press, New Brunswick. New Jersey, 1983) subrayó la «modernidad» de los elementos más extravagantes de la religiosidad decimonónica francesa.

${ }^{68}$ Resaltada, de manera muy particular, en los trabajos de Manuel Suárez Cortina. Así, en el último incluye el krauso-institucionismo, junto al espiritismo, en el apartado dedicado a las religiosidades alternativas: Entre cirios y garrotes. Política y religión en la España contemporánea, 1808-1936, Universidad de Cantabria-Universidad de Castilla-La Mancha, Santander-Cuenca, 2014, pp. 187-222.

${ }^{69}$ El género biográfico será de gran utilidad para hacernos ver esa diversidad en el seno de una confesión. Un ejemplo reciente en Feliciano Montero García, Antonio C. Moreno Cantano, Marisa Tezanos Gandarillas (coords.), Otra Iglesia. Clero disidente durante la Segunda República y la guerra civil, Trea, Gijón, 2013. 
miembros de una sociedad moderna, sean creyentes o no ${ }^{70}$, son factores que deberá tener en cuenta el historiador cuando intente comprender el modo de actuar en sociedad de personas y colectividades que se identifican como religiosos.

Al menos, dos asuntos más demandarían la sutileza de los historiadores a la hora de interpretar la complejidad de las relaciones entre religión y modernidad. El primero - en una vía de investigación que, cuando se ha abordado, se ha demostrado fructífera - remitiría a la consideración de las estrategias modernizadoras adoptadas por parte de las confesiones religiosas o por grupos de creyentes. En unas ocasiones, la «modernización» religiosa reflejará una aspiración sincera a avenirse con la modernidad «secular». En la mayoría de los casos, sin embargo, probablemente expresará un no menos sincero deseo de formular una modernidad propia, que puede adquirir ciertos rasgos - si bien, no necesariamente - de «modernidad defensiva» ${ }^{71}$. El segundo asunto al que quisiera referirme resultará seguramente más familiar al conjunto de la profesión historiográfica. Es imposible darle siquiera un mínimo desarrollo aquí, pero la ausencia de, al menos, su mención estaría totalmente injustificada. Me refiero al estudio de las formas de «sacralidad fuera de la religión» y su competencia con la sacralidad religiosa más tradicional ${ }^{72}$. Estas formulaciones se desarrollaron a lo largo del siglo XIX en forma de religiones seculares, desde el positivismo al nacionalismo, y en los años veinte y treinta del siglo XX, en forma de religiones políticas. Una breve referencia a la cuestión del nacionalismo nos llevaría a resaltar que la religión, y más concretamente el catolicismo, está pasando de ser considerada por politólogos e historiadores como un elemento retardatario de los proce-

70 De nuevo me remito a Berger, The Many Altars, op. cit., pp. X-XI y 51-67, pero también a Taylor, A Secular Age, op. cit., pp. 1-10 y 539-593.

${ }^{71}$ Un término introducido entre nosotros por Francisco Javier Caspistegui, «El cine como instrumento de modernidad defensiva en Pamplona, 1917-1931», Ikusgaiak, 7, 2005, pp. 5-38. Una reflexión sobre la «modernidad religiosa» en Francisco Javier Ramón Solans, La Virgen del Pilar dice... Usos nacionales de un uso mariano en la España contemporánea, PUZ, Zaragoza, 2014, pp. 17-25. La capacidad modernizadora de grupos religiosos católicos en Maitane Ostolaza Esnal, Entre religión y modernidad. Los colegios de las congregaciones religiosas en la construcción de la sociedad guipuzcoana contemporánea, 1876-1931, Universidad del País Vasco, Bilbao, 2000.

72 Françoise Champion, Sophie Nizard y Paul Zawadki, «Reformuler la question du sacré en modernité», en Françoise Champion, Sophie Nizard y Paul Zawadzki (eds.), Le sacré hors religions, L'Harmattan, París, 2007, pp. 9-22. 
sos de construcción nacional a valorarse positivamente su contribución a estos. Una más breve referencia aún a las religiones políticas nos llevaría a destacar la polémica, y al tiempo fructífera, recepción de este concepto en nuestra historiografía nacional ${ }^{73}$.

A modo de conclusión, cabría de nuevo preguntarse si resulta todavía válido manejar el concepto de secularización en el ámbito de la historia. La opinión de quien suscribe estas líneas es que sí, que es todavía válido o que al menos, como proponía Owen Chadwick hace cuarenta años - cuando ya se oían las primeras críticas sobre su pertinencia-, todavía es un concepto «útil»» ${ }^{74}$. Un concepto útil, siempre y cuando se parta de la base de su inherente «conflictividad». La propuesta historiográfica que aquí se defiende sería, pues, la de una «secularización conflictiva» ${ }^{75}$. «Conflictiva» en una doble dirección. En una primera, sería conflictiva porque lo es en el presente debate científico sobre el paradigma al que da nombre. A la vista de este debate, ya no caben las asunciones clásicas sobre la universalidad e inexorabilidad del proceso de secularización y el declive de la religión. Sólo desde una problematización del concepto de secularización puede su uso continuar siendo plausible. En una segunda dirección, la secularización sería conflictiva porque no cabe duda de que su desarrollo lo fue en el pasado. El papel de la religión en la esfera pública $-\mathrm{y}$ hasta en la privada - ha sido objeto permanente de polémica y enfrentamiento, de conflicto en el plano político y cultural. Su pérdida de pertinencia social en la modernidad rara vez ha sido un proceso gradual, lento y tranquilo. Normalmente ha sido el resultado de una confrontación entre fuerzas confesionales y secularizadoras. La secularización bien ha podido ser un «mito», como

73 Joseba Louzao, «Nación y catolicismo en la España contemporánea. Revisitando una interrelación histórica», Ayer, 90 (2013), pp. 65-89; Alfonso Botti, Feliciano Montero y Alejandro Quiroga (coords.), Católicos y patriotas. Religión y nación en la Europa de entreguerras, Sílex, Madrid, 2013; Ramón Solans, La Virgen del Pilar dice..., op . cit.; Zira Box, «La tesis de la religión política y sus críticos: aproximación a un debate actual», Ayer, 62, 2006, pp. 195-230; Pedro Carlos González Cuevas, «Las religiones políticas contemporáneas: su incidencia en España», en Julio de la Cueva y Feliciano Montero (eds.), Laicismo y catolicismo. El conflicto político-religioso en la Segunda República, Alcalá de Henares, Universidad de Alcalá, 2009, pp. 91- 126.

${ }^{74}$ Owen Chadwick, The Secularization of the European Mind in the Nineteenth Century, Canto, Cambridge, 1975, pp. 2-3.

${ }_{75}$ Y que dio título al libro de Julio de la Cueva y Feliciano Montero (eds.), La secularización conflictiva. España, 1898-1931, Biblioteca Nueva, Madrid, 2007. 
han denunciado algunos sociólogos ${ }^{76}$; pero ha sido, en cualquier caso, un mito «verdadero» 77 , un mito genuino en cuanto ha proporcionado un relato intensamente movilizador a favor y en contra. También los historiadores estamos llamados a desentrañar sus diversos argumentos y los conflictos que ha generado.

76 Peter Glasner, The Sociology of Secularization. The Critique of a Concept, Routledge, Londres, 1977; Joan Estruch, «El mito de la secularización», en Rafael Díaz-Salazar, Salvador Giner y Fernando Velasco (eds.), Formas modernas de religión, Alianza, Madrid, 1994, pp. 267-280; Cox, «The Myth», op. cit.

77 En algunos casos, como el europeo, puede que incluso una auténtica profecía autocumplida (Casanova, «Beyond European», op. cit.). 